\title{
TEMPORALIDAD Y CONDUCTA SUICIDA
}

\section{López Rodríguez, Juan Luís* Bárcena Calvo, Carmen** González Medrano, Julia*** Iglesias Guerra, José Antonio**** Abella García, Víctor***** \\ * Doctor. Profesor Asociado en la E.U.E de Oviedo. \\ ** Doctora. Profesora Titular de la E.U. de Ciencias \\ de la Salud de la Universidad de León. \\ *** Diplomada en Enfermería.}

**** Licenciado en Filosofía y Ciencias de la Educación. Profesor Asociado de la

E.U. de Ciencias de la Salud de la Universidad de León.

***** Doctor. Profesor Asociado de la Universidad de Burgos.

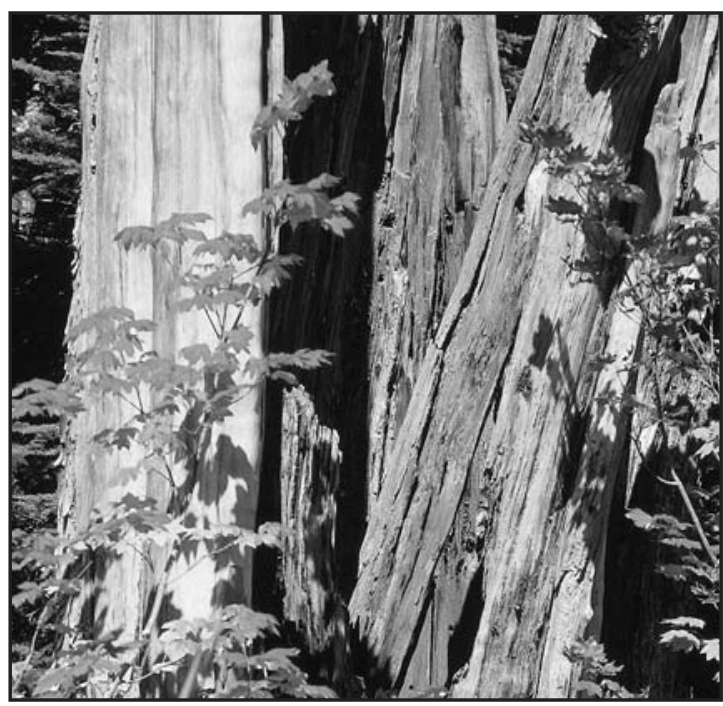

TEMPORALITY AND SUICIDAL BEHAVIOUR

\section{SUMMARY}

Objetive: To evaluate the temporality and the psicosocial profile of some suicidal behaviors that were taken care of in the Asturias Central University Hospital (HUCA)

Design: Prospective study

Patients: 1633 subjects, who were assisted in the Emergency Unit due to a suicide attempt, were studied in 2003-2005.

Results: The subjects who have had some kind of suicidal behavior are usually 30-39 years old, female, single, living with their family, with primary education, unemployed, and of Spanish nacionality. In regard to the temporality pattern, suicide attempts are commited in the evening, during weekdays, and in summer time.

Key words: Sicide attempt, suicidal behaviour, epidemiology in a Universiy Hospital.

\section{TEMPORALIDADE E CONDUTA SUICIDA}

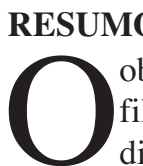
objetivo é avaliar a temporalidade e o perfil psico-social das condutas suicidas atendidas no Hospital Universitário Central de Astúrias (HUCA). O estudo tem um desenho prospectivo. Os sujeitos do estudo foram 1633 pacientes, que recorreram ao Serviço de Urgências por apresentar conduta suicida, durante o triênio de 2003 a 2005. Os resultados mostraram que os sujeitos que desenvolveram conduta desse tipo, têm como perfil o fato de pertencerem ao grupo etário compreendido entre 30 e 39 anos; mulher, solteira, que convive com família própria, com estudos primários, inativa do ponto de vista econômica e trabalhista, e de nacionalidade espanhola. Em relação ao padrão de temporalidade, as tentativas de suicídio caracterizam-se por serem concretizadas durante o período vespertino, em dias de semana, e durante os meses de verão.

Palavras-chave: Tentativa de suicídio; Conduta suicida; Epidemiologia em um Hospital Universitário. 


\section{RESUMEN}

Objetivo: Evaluar la temporalidad y el perfil psicosocial de las conductas suicidas atendidas en el Hospital Universitario Central de Asturias (HUCA).

Diseño: Estudio prospectivo.

Pacientes: Fueron estudiados 1.633 sujetos que acudieron al Servicio de Urgencias por presentar conducta suicida durante el trienio 2003-2005.

Resultados: Los sujetos que han realizado algún tipo de conducta suicida, se caracterizan por pertenecer al grupo de edad comprendido entre los 30 y 39 años, mujer, soltera, que convive con familia propia, con estudios primarios, inactiva económica y laboralmente, y de nacionalidad española. En relación al patrón de temporalidad, las tentativas de suicidio se caracterizan por ser llevadas a cabo durante las horas de la tarde, en días de semana, y durante los meses estivales.

Palabras clave: Tentativa de suicidio, Conducta suicida, Epidemiología en un Hospital Universitario.

\section{INTRODUCCIÓN}

Dentro de los numerosos acontecimientos enigmáticos que aporta la vida humana, la conducta suicida es uno de ellos. Son inciertas, hasta el momento, las razones por las cuáles una persona atenta contra su vida, cuando su más preciado tesoro es la vida misma. La conducta suicida, como fenómeno, abarca el intento o tentativa y el suicidio, este último, particularmente, con una rica historia (Gómez et al 1996).

Las tentativas de suicidio son el factor de riesgo más importante para el suicidio consumado. La OMS destaca la importancia de los comportamientos suicidas no letales como un grave problema de Salud Pública. Así pues, tanto la valoración como el tratamiento de este tipo de conductas en los servicios de urgencias van a resultar factores clave en el tratamiento de las personas con conductas suicidas y potencialmente en la prevención del suicidio.

Se trata de uno de los principales problemas sanitarios a pesar de que la mortalidad por suicidio en población general es relativamente baja (1\%$2 \%$ de la mortalidad por cualquier causa) y ocupa entre la $9^{\mathrm{a}}$ y $10^{\mathrm{a}}$ posición en las causas de mortalidad en los países occidentales, existiendo en España una incidencia de tentativas suicidas aproximadamente de entre 30.000 / 40.000 personas/año. Esto vendría a suponer unas tasas de entre 50 y 90/100.000 habitantes/año, aunque las tasas reales probablemente sean superiores (Saiz, 1997).

\section{MATERIAL Y MÉTODO}

Se realiza un estudio prospectivo con una muestra constituida por 1.633 sujetos que acudieron a los Servicios de Urgencias del Hospital Universitario Central de Asturias (HUCA), sito en la ciudad de Oviedo, durante el período comprendido entre el 1 de Enero de 2003 y el 31 de Diciembre de 2005. A todos los sujetos se les pasó, como instrumento de evaluación, el WHO/EURO MULTICENTRE STUDY OF SUICIDAL BEHAVIOUR MONITORING FORM (Schmidtke, et al 1996).

Se trata de un protocolo constituido por un total de 29 variables de carácter cuantitativo y que recogen información acerca de:
A.- Datos sociodemográficos:
B.- Tentativa de suicidio actual:
C.- Tentativas previas:
D.- Diagnósticos psiquiátricos:
E.- Tratamiento actual en algún dispositivo de la Red de Salud Mental:

\section{RESULTADOS}

El total de sujetos que acudieron durante ese período de tiempo a los Servicios de Urgencias fue de 259.293 sujetos, de los cuáles, 1.633 (0,63\%) lo hicieron por presentar algún tipo de conducta suicida.

La población muestral estaba compuesta por 636 varones $(38,9 \%)$ y 997 mujeres $(61,1 \%)$. La proporción de hombres que han realizado tentativa suicida es inferior a la proporción de hombres en la población general del Área Sanitaria. En las mujeres evidentemente sucede lo contrario. No obstante, las diferencias no son estadísticamente significativas. La edad media [media (desviación estándar)] de la muestra fue de [38,66 $(15,13)]$ años de edad, siendo la edad mínima de 13 y la máxima de 95 años. En los hombres predominan las edades comprendidas entre los 30 y los 39 años 
de edad seguido del grupo de 20 a 29 años de edad, mientras que en el grupo de las mujeres, predominan en primer lugar las edades comprendidas entre los 30 y los 39 años de edad pero son seguidas del grupo de 40 a 49 años de edad (Tabla 1).

\begin{tabular}{|l|l|l|l}
\hline $\begin{array}{l}\text { Grupos de edad } \\
{[N(\%)]}\end{array}$ & Hombres & Mujeres & Total \\
\hline $\begin{array}{l}13-19 \text { años } \\
20-29 \text { años }\end{array}$ & $32(5)$ & $73(7,3)$ & $105(6,4)$ \\
$30-39$ años & $149(23,4)$ & $219(22)$ & $368(22,5)$ \\
$40-49$ años & $213(33,5)$ & $266(26,7)$ & $479(29,3)$ \\
$50-59$ años & $136(21,4)$ & $233(23,4)$ & $369(22,6)$ \\
$60-69$ años & $46(7,2)$ & $114(11,4)$ & $160(9,8)$ \\
$70-79$ años & $24(3,8)$ & $36(3,6)$ & $60(3,7)$ \\
$80-89$ años & $20(3,1)$ & $29(2,9)$ & $49(3)$ \\
$\geq 90$ años & $12(1,9)$ & $20(2)$ & $32(2)$ \\
\hline Dato desconocido & $1(0,2)$ & $5(0,5)$ & $6(0,4)$ \\
\hline
\end{tabular}

La distribución según estado civil fue de solteros $(39,8 \%)$, casados o con pareja estable $(31,2 \%)$, viudos, divorciados, y separados $(4 \%, 3 \%, \mathrm{y} 10 \%)$ respectivamente. En relación al soporte familiar, $154(9,4 \%)$ sujetos vivían solos, 94 (5,8\%) vivían con sus hijos, $468(28,7 \%)$ vivían con pareja e hijos, $117(7,2 \%)$ con pareja sin hijos, 457 (28\%) vivían con su familia de origen, $145(8,8 \%)$ vivían con otras personas y/o en Instituciones. A nivel académico se encontró que predominan los sujetos con estudios primarios 1062 (65\%), seguidos de los sujetos con educación secundaria $276(16,9 \%)$ y de los sujetos con estudios universitarios 99 $(6,1 \%)$. La situación laboral se distribuyó de la siguiente forma: $628(38,5 \%)$ se encontraban en activo laboralmente, $268(16,4 \%)$ percibían algún tipo de prestación económica aunque sin trabajo activo, y $552(33,8 \%)$ no recibían prestaciones económicas ni desarrollaban trabajos remunerados (amas de casa, estudiantes, etc.). Con respecto a la nacionalidad de los sujetos, prácticamente la totalidad de la muestra 1.565 (96\%) tenían nacionalidad española y 66 (4\%) eran extranjeros.

Con relación a la temporalidad de las tentativas de suicidio, en el presente estudio se han realizado con mayor frecuencia durante las horas 23 $(7,5 \%)$, seguidas de las horas $12(7,4 \%), 20$ $(6,9 \%)$, las $21(6,5 \%)$, las $19(5,8 \%)$, las $17(5,7 \%)$ y las $22(5,5 \%)$. La mayoría de las tentativas se llevan a cabo entre las 12 y las 0 horas. En función del día de la semana, $898(55,1 \%)$ de los sujetos lo hicieron durante los días de semana (lunes, martes, miércoles y jueves), en tanto que $733(44,9 \%)$ eligieron el fin de semana (viernes, sábado y domingo). Los días de la semana en que se produce un mayor número de tentativas de suicidio han sido los domingos $(16,9 \%)$ y $\operatorname{los}$ lunes $(15,2 \%)$ respectivamente. Los días en que menos tentativas se han producido han sido los miércoles $(12,9 \%)$. Con respecto al mes, 376 $(23,1 \%)$ realizaron la tentativa de suicidio durante el primer trimestre, $416 \quad(25,5 \%)$ lo hicieron durante el segundo trimestre, $458 \quad(28,1 \%)$ lo hicieron durante el tercer trimestre y $383(23,5 \%)$ lo hicieron durante el cuarto trimestre. El procedimiento empleado con mayor frecuencia para las tentativas de suicidio fue la intoxicación medicamentosa, elegida por el $(74,8 \%)$ de los sujetos, seguido por la flebotomía $(18,4 \%)$ de los sujetos. Con una menor frecuencia la precipitación (3\%), la ingestión de pesticidas y ahorcamiento $(1 \%)$.

\section{DISCUSIÓN}

En la mayor parte de los países donde se ha estudiado el fenómeno suicida, las mujeres presentan mayores tasas de ideación y de comportamientos suicidas (Kopjar et al 2005; Agudo et al 2006; Duce et al 1998), pero la mortalidad producida por estos actos es típicamente más baja en las mujeres que en los varones.

En España, la proporción entre mujeres y varones por lo que se refiere a tentativas de suicidio, presenta una tendencia hacia la equiparación. Así, se ha pasado de una relación de 1:9 a favor de las mujeres en la década de los 70 a 1:2 en la actualidad. En la muestra estudiada el número de mujeres que realizaron una tentativa suicida fue de 997 $(61,1 \%)$ en tanto que los varones fueron 636 $(38,9 \%)$. Los porcentajes de hombres y mujeres del presente estudio, son coincidentes con los mismos en población general, siendo la proporción mujer/hombre de 1,6. Los grupos de edad más frecuentemente relacionados con las tentativas de suicidio son los incluidos en el período juvenil. La mayoría de los estudios que investigan este fenó- 
meno, muestran cómo las tasas más elevadas de comportamiento parasuicida se dan en personas jóvenes, concretamente en la franja de edad comprendida entre los 15 y los 34 años (Fernández, 2001; Lozano et al 2004; Bille-Brahe et al 1997).

En el estudio WHO/EURO se observó que la relación entre intentos de suicidio y estado civil era dependiente de la edad por cuanto la probabilidad de estar casado es menor entre la población más joven. Aún así, al corregir este factor de confusión se observó que las tentativas suicidas eran más frecuentes entre los solteros y los divorciados. Por último, se considera que tener hijos a cargo es un factor de protección frente a este tipo de comportamientos (Bobes et al 2004)). La situación psicológica de aislamiento (alejamiento conyugal, migración, etc.) aumenta el riesgo de tentativa suicida. Así, circunstancias como vivir sólo, estar ingresado en un hospital, en la cárcel, etc. elevarían las posibilidades de realizar este tipo de conducta.

Existen datos consistentes de que la disfunción familiar podría ser un factor favorecedor del comportamiento suicida. Un estudio epidemiológico que ha evaluado a 12.500 sujetos de cinco países europeos (Reino Unido, Irlanda, España, Noruega y Finlandia) señala el aislamiento como uno de los factores asociados a la presencia de ideas suicidas en la población que se repite en todos los países europeos (Ayuso et al 2006). En general, se considera que el nivel educativo no tiene un papel determinante en el comportamiento suicida. No obstante, lo cierto es que en la mayoría de los estudios se encuentra un nivel educativo más bajo en las personas que intentan o consuman el suicidio (Caracciolo, 1996). En los datos encontrados en el presente estudio, los sujetos con estudios primarios están más representados que en la población general, obteniéndose diferencias estadísticamente significativas tanto en hombres como en mujeres.

La relación entre conducta suicida y desempleo ha sido comprobada en numerosas ocasiones, coincidiendo en señalar la existencia de una relación entre las conductas suicidas y diversos marcadores socioeconómicos, entre los que destaca el desempleo, la duración del mismo o la clase social de pertenencia . A pesar de que el desempleo se asocia a las tentativas de suicidio serias tanto en hombres como en mujeres, el riesgo atribuible es sólo del 7,3\%, lo que sugiere que la situación de desem- pleo contribuye en una pequeña medida al desencadenamiento del acto suicida, siendo por tanto fuente de controversia como factor de riesgo. El lugar de nacimiento del individuo viene a demostrar de una manera "incorrecta" el reflejo de la emigración. La inmigración, unida al desarraigo y el aislamiento, supone un factor de riesgo de comportamientos suicidas, aunque este riesgo disminuye cuando la emigración se hace con toda la familia. Esto se ha observado en las familias de emigrantes en las que se ha mantenido la misma tasa de suicidio de su país de origen durante las primeras generaciones, y parece apoyar la teoría de la integración social de Durheim, ya que la mayor capacidad de adaptación protege frente a este efecto suicidógeno de la emigración.

La franja horaria correspondiente a la tarde y noche, con independencia de la gravedad de la tentativa, del género, o de la repetición, es mayoritariamente la más prevalerte en las tentativas de suicidio, y la preferida sobre todo en los Trastornos Mentales Orgánicos, sujetos dependientes de sustancias psicoactivas, Trastornos Neuróticos y de la Personalidad. Algunos autores sugieren que en el horario pueden influir posiblemente los ritmos circadianos biológicos, puesto que en las horas de la tarde y primeras de la noche, los niveles de serotonina están más bajos (Jessen et al 1999). Los días de la semana son objeto de discusión entre los investigadores, ya que tanto en el estudio multicéntrico europeo (Schmidtke et al 1996) como en otras culturas (Ho, 1998), encuentran, para las tentativas, los domingos como el día con el mayor pico, en tanto que los viernes sería el más bajo, aunque esto, sólo sería válido para las mujeres, concentrándose después de días vacacionales señalados, como la Navidad, y no antes. Las variaciones estacionales en las tentativas de suicidio se han divulgado en muchos países. Investigaciones recientes han sugerido que los patrones estacionales entre las tentativas y los suicidios consumados no son iguales. Para la mayoría de los investigadores que estudian las tentativas de suicidio, éstas son más bajas durante los meses de invierno, incrementándose hasta la primavera y con picos en mayo y junio.

En otras culturas los picos de tentativas que ingresan en hospitales (excluyen intoxicaciones por alcohol) se ven durante los meses de junio, 
agosto y septiembre, relacionando estos dos últimos meses con factores culturales-religiosos, ya que las fiestas serían factores de riesgo para las sociedades con poco soporte familiar y social, mientras que resultarían protectoras donde existe dicho soporte, puesto que favorecerían la reunión con los suyos. Los métodos utilizados en las tentativas de suicidio suelen ser métodos "blandos" (sobredosis de fármacos y cortes o incisiones en las flexuras de las manos), lo que indicaría la ambivalencia existente en esos momentos.

\section{BIBLIOGRAFÍA}

- Agudo, M. et al. (2006) Conductas parasuicidas en población inmigrante del área 11 de salud; X Congreso Nacional de Psiquiatría.

- Ayuso Mateos, JL. et al. (2006) Predictors of compilance with psychosocial interventions offered in the community; Psychological Medicine, 1: 1-9.

- Bille-Brahe, U. et al. (1997) A repetition-prediction study of european parasuicide populations: a summary of the first report from part II of the WHO/EURO multicentre study on parasuicide in cooperation with the EC Concerted Action on Attempted Suicide; Acta Psychiatr.Scand, 95: 81-86.

- Bobes, J, Sáiz, P, Bascarán, MT, Bousoño, M, y GarcíaPortilla, MP (2004). Comportamientos suicidas. Prevención y tratamiento. Barcelona: Ars Médica.

- Caracciolo, S. (1996) Circadian rhythm of parasuicide in rela- tion to violence if method and concomitant mental disorder. Acta Psychiatrica Scandinavica, 93(4): 252 - 256.

- Duce, S. et al. (1998) Intoxicaciones medicamentosas voluntarias atendidas en un servicio de urgencias. Emergencias, 10(4): 225-233

- Fernández, C. (2001) Características de las conductas suicidas en pacientes ingresados en una unidad psiquiátrica. Universidad de Oviedo, Tesis Doctoral.

- Gómez Alcalde, M.S.; Reyes Martín, A. (1996) Suicidio consumado y enfermedad mental previa: aproximación epidemiológica. Psiquis, 17(8): 402-409.

- Ho, B (1998) Temporal variation in parasuicide among Singaporean Chinese. Australian and New Zaeland. Journal of Psychiatry, 32: 500-503.

- Jessen, G. et al. (1999) Temporal fluctuations and seasonality in attempted suicide in Europe. Archives of Suicide Research, 5: 57-69.

- Kopjar, B.; Dieserud, G.; WilK, J. (2005); Deliberate self-poisonings treated in hospitals. Tidsskr. Nor. Laegeforen. 125(13):1798-800

- Lozano, C. et al. (2004) Estudio descriptivo del parasuicidio en las urgencias psiquiátricas. Rev. Asoc. Esp Neuro. 91:1122.

- Saiz Martinez, P.A. et al. (1997); Searching for a predictive peripherical biological model in parasuicidal behaviour. Eur Psychiatry, 12: 75-81.

- Schmidtke, A et al. (1996) Attempeted suicide in Europe: rate, trendes and sociodemographic characteristics of suicide attempters during the period 1989-1992. Results of the QWHO/EURO multicentre study on parasuicide. Acta Psychiatric Scan, 327-338.

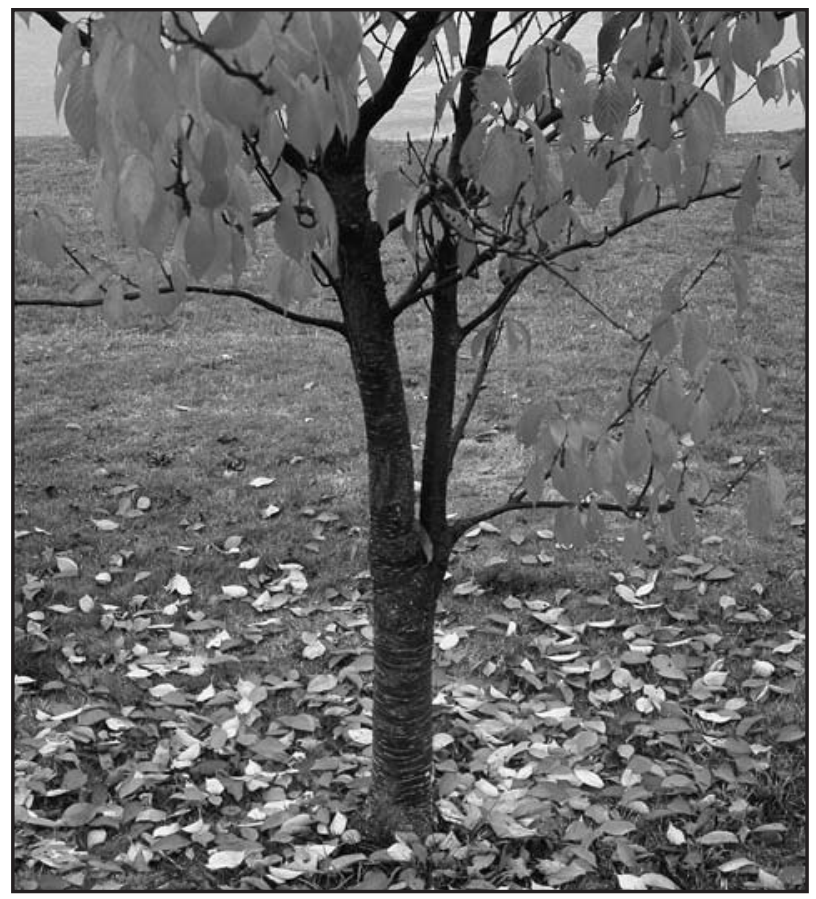

